\title{
Dieux et défunts au Japon : La Grande Bouffe
}

Jean-Pierre GIRAUD

\section{OpenEdition}

Journals

Édition électronique

URL : http://journals.openedition.org/transtexts/601

DOI : 10.4000/transtexts.601

ISSN : 2105-2549

Éditeur

Gregory B. Lee

Référence électronique

Jean-Pierre GIRAUD, «Dieux et défunts au Japon : La Grande Bouffe», Transtext(e)s Transcultures 跨文 本跨文化 [En ligne], 10 | 2015, mis en ligne le 21 juin 2016, consulté le 19 avril 2019. URL : http:// journals.openedition.org/transtexts/601; DOI : 10.4000/transtexts.601

Ce document a été généré automatiquement le 19 avril 2019

(c) Tous droits réservés 


\title{
Dieux et défunts au Japon : La Grande Bouffe
}

\author{
Jean-Pierre GIRAUD
}

1 S'il est bien connu que les vivants aiment se délecter des plaisirs de la table (jusqu'à s'empiffrer d'ailleurs), il apparaît, comme nous allons le voir, qu'il en est de même des dieux comme des défunts japonais.

2 En effet, les offrandes alimentaires dans les temples shintoïstes, à l'usage des dieux et des déesses ou dans les monastères bouddhistes à l'usage des bouddhas et des boddhisattvas, ou encore celles que l'on destine aux cultes funéraires - offrandes et banquets libératoires - sont à la fois très présentes et essentielles pour accomplir les rites. En outre, ces mêmes offrandes alimentaires et les banquets qui leurs sont associés revêtent un caractère psychopompe tout à fait évident dans l'imaginaire nippon.

3 Mais y aurait-il un imaginaire sans la mort et son traitement rituel ?

4 C'est pourquoi le clin d'œil quelque peu iconoclaste du titre au célèbre film La Grande Bouffe de Marco Ferreri en vient à poser les bases de notre réflexion d'aujourd'hui.

5 Les espaces sacrés réservés aux dieux, aux bouddhas ou aux défunts (qui sont parfois les mêmes, voire voisins) vont rarement sans offrandes sur des plateaux rituels de riz, de sel et de saké. A ces offrandes de base sont parfois ajoutés des mandarines, des mochi ${ }^{1}$ voire d'autres aliments.

6 Or, de même que l'on présente ainsi aux dieux leur auguste repas, le défunt reçoit le sien, le jour de son trépas. Celui-ci est constitué d'un bol de riz blanc (sen mai) ou d'un plateau repas simple (jôsen). Un banquet est également organisé à proximité du mort pour la veillée ${ }^{2}$ funéraire (o tsûya) au domicile.

7 Tous ces rites perdurent de nos jours, certes de façon souvent atténuée. On les pratique par «tradition ", par habitude, mais sans trop savoir, sans trop comprendre les pratiques et sans prendre conscience de leur double rattachement dans l'histoire de l'imaginaire cultuel du Japon ancien. 
8 D'un point de vue strictement historique, c'est dans l'ancien rite du môgari impérial (c'està-dire la gestion de la période de deuil à l'usage de la mesnée impériale et plus spécifiquement relatif aux funérailles des empereurs) qu'on peut faire remonter la codification de ces rites funéraires complexes.

9 Le môgari n'était bien sûr pas uniquement constitué par des offrandes alimentaires ou des banquets, puisque bien d'autres rites y étaient rattachés. Les funérailles de l'Empereur Temmu (622 - 686), auquel succédera sur le trône l'Impératrice Jitô (645 - 703), sont bien détaillées dans les récits que nous en donnent le Kojiki ${ }^{3}$ et le Nihonshoki ${ }^{4}$, entres autres ${ }^{5}$.

Pourtant, si l'on peut affirmer que c'est avec Temmu que ces rites se sont peu à peu dogmatisés (jusqu'à disparaître d'ailleurs, peu de temps après, avec la disparition du môgari), ils se retrouvent d'une façon plus ou moins directe dans les mythes japonais qui nous relatent des événements centrés sur l'époque de Yayoi (soit une période qui va du 3ème siècle avant notre ère au 3ème siècle après notre ère). Ces mythes, pour certains, prennent eux-mêmes racine dans des pratiques rituelles plus anciennes, remontant au néolithique, voire au paléolithique japonais, sans pour autant que l'on puisse dater cela avec certitude.

11 C'est donc dans ces mythes que l'article se propose de tenter de découvrir les origines de l'alimentation et, dans une seconde partie, de discuter du mythème du banquet, en gardant bien en mémoire que l'alimentation des dieux comme le banquet véhiculent tous deux une très forte connotation psychopompe.

Les divinités des céréales :

Selon le Kojiki, le dieu Izanagi donne naissance à trois divinités : Amaterasu oho mikami (divinité solaire, ancêtre de la mesnée impériale), Take haya susanoo no mikoto (divinité terrestre à fonctions multiples et qui va particulièrement nous intéresser) et Tsukuyomi no kami (une divinité de la lune, qui forme un couple avec la précédente).

Prenant racine dans les commentaires des Kokugakusha de l'époque d'Edo tels que Motoori Norinaga, Kamo no Mabuchi ou Hirata Atsutane, la plupart des mythologues voient encore ce dieu Susanoo et les deux mythes qui suivent sa naissance ${ }^{6}$ de façon uniquement manichéenne, le dieu s'opposant à sa sœur le soleil, commettant des exactions inondations, magie noire, provoquant la mort de la déesse ... Or cette lecture paraît trop simpliste pour rendre compte de la valeur et de la fonction du mythe. Toute notion de conflit ou de sacrifice est jugée comme étant un mal lorsque ces mêmes éléments sont, à l'évidence, des actes nécessaires et créateurs qui permettent justement la suite du récit mythique.

15 Ce mythe présente d'ailleurs une fonction calendaire très précise, qui rythme le récit selon l'année rizicole. Ainsi, les actes du dieu deviennent nécessaires et compréhensibles : lorsqu'il brise les digues et provoque des inondations, c'est pour figurer le rite de ta ue qui consiste à inonder les rizières pour mettre en eau les jeunes plants; lorsqu'il pratique la magie noire (présentation d'un cheval écorché à rebours), c'est un rite de remerciement pour célébrer la récolte; lorsque son acte fait mourir une desservante d'Amaterasu (qui préfigure Amaterasu elle-même), c'est pour faire mourir le soleil (au solstice), afin qu'il puisse renaître.

16 Allant plus avant dans cette lecture des mythes concernés, nous ne pouvons pas ne pas remarquer que le dieu Susanoo accomplit son destin au centre d'un vaste système lié à l'agriculture, ainsi qu'à la nourriture qui en découle. Mais plus que cela encore, les ascendants comme les descendants du dieu s'inscrivent eux aussi dans le même système. 
17 Rien d'étonnant au Japon, où l'habitude des récits à tiroir, où l'on vous raconte plusieurs fois de suite la même chose, où les textes sont sans arrêt en relation avec d'autres par des liens intertextuels et des images palimpsestes, est profondément ancrée.

Chassé du ciel à l'issue du mythe de la caverne, Susanoo redescend sur terre et rencontre en chemin une déesse nommée Oho getsu hime no kami. Alors que son nom même comporte la racine " ge » (ke / ge / uke / uka et mike), c'est très clairement une divinité en rapport avec les aliments. Elle offre d'ailleurs à Susanoo des mets préparés qui proviennent de sa bouche, de son nez, de son prôktos ... Comme le dieu pense alors que ceux-ci sont impurs, il tue la déesse, du corps de laquelle vont apparaître : le vers à soie (provenant de la tête), le riz (des yeux), le millet (des oreilles), les haricots rouges (du nez), le blé (du sexe) et les haricots (de son anus) ${ }^{7}$.

Il nous est dit ensuite que la divinité de l'énergie Kamu musuhi no kami ramasse les différentes graines et s'en sert comme semences (pour produire la nourriture nécessaire).

Le meurtre sacrificiel de la déesse Oho getsu hime est donc bien nécessaire et créateur et il en découle que ce récit, plus encore que le précédent (avec son cycle calendaire de la riziculture) place Susanoo en tant que dieu tutélaire des aliments et de leur production.

21 Or, si l'on cherche à convoquer la présence des divinités liées aux céréales (ou à la nourriture) dans le Kojiki, on bute indubitablement sur deux épisodes qui sont en amont, puis en aval, des récits mettant en scène directement Susanoo.

Pour le premier épisode, il s'agit des kami qui apparaissent lors de l'agonie d'Izanami no kami (provoquée par la naissance de son fils Kagutsuchi no kami, dieu du feu) ${ }^{8}$; or, la déesse Izanami peut être vue comme la mère de Susanoo.

Quant au second épisode, il nous renvoie aux kami qui proviennent de la hiérogamie entre Susanoo et Kamu oho ichihime no kami, dans le mythe qui suit directement le meurtre sacrificiel de Oho getsu hime.

Dans le premier cas, Izanami, lors de son agonie, donne naissance à des divinités de la mine, puis de la poterie et ce, par ses vomissures, puis par ses excréments. De son urine apparaît ensuite une divinité de l'eau Mitsu ha no me no kami (qui transcende l'eau nécessaire pour l'agriculture), ainsi qu'une divinité jeune de la croissance qui est clairement une divinité agricole et qui aura pour fille Toyo uke bime no kami.

Or, cette dernière est assimilable à Oho getsu hime par sa fonction, si ce n'est qu'elle conservera d'ailleurs cette fonction dans les rituels shintô à venir (alors que Oho getsu hime n'existe que dans les mythes). Toyo uke bime deviendra en effet une divinité fort importante dans les rituels, dès l'époque de l'empereur Tenmu, puisque l'on va dès lors l'intégrer au centre des croyances et pratiques cultuelles d'Ise (donc des rituels essentiels liés à la famille impériale). Ainsi Amaterasu sera-t-elle sanctifiée dans le naigû (le Temple intérieur d'Ise, le principal) et Toyo uke le sera dans le gegû (le Temple extérieur d'Ise). ${ }^{9}$

Pour ce qui est du second épisode qui nous intéresse, il a lieu après que Susanoo a accompli le meurtre rituel de la déesse Oho getsu hime. Susanoo arrive dans le pays d'Izumo (qui, pour l'imaginaire japonais, est plutôt une contrée liée à la mort ${ }^{10}$ ) et il se retrouve confronté à un rituel de sacrifice d'une jeune vierge céleste à une divinité terrestre, représentée par un serpent à 8 têtes et 8 queues nommé Yamata no orochi. Susanoo tue par ruse le serpent et met ainsi fin à cette pratique calendaire d'offrande de sacrifice $^{11}$. Là encore, c'est le motif manichéen qui a été retenu de prime abord, puis exploité à l'envi. Susanoo semble certes mettre dans ce mythe sa violence au service du 
bien, mais il continue aussi de montrer sa violence intrinsèque, donc son caractère premier, qui est négatif.

Pourtant, une lecture palimpseste du texte nous montre facilement que les choses ne sont pas si simples que cela, puisque le dieu (qui a déjà pour fonction première, et comme nous l'avons montré, d'être une divinité tutélaire de l'agriculture) sauve du sacrifice Kushi inada hime qui est une divinité à la fois de la rizière (inada) et à la fois du serpent (kushi désigne certes le peigne, mais la forme du peigne nous renvoie à la tête du serpent). En outre, les parents de la divinité qui se nomment Ashi na zuchi et Te na zuchi (l'Esprit sans jambe et l'Esprit sans bras) sont clairement des serpents. La divinité (serpent-rizière) offerte en sacrifice doit être dévorée par un serpent, le dieu terrestre Yamata no orochi.

Or, on peut affirmer que ce Yamata no orochi est, et préfigure, le dieu agricole des rituels en devenir, soit le dieu de la montagne (Yama no kami) qui se change en dieu des champs (Ta no kami) le printemps venu. Ce dieu serpent qui va protéger la terre (les rizières) et donc la féconder symboliquement, pour engendrer la récolte.

Le sacrifice (meurtre rituel) du dieu terrestre est là encore un acte nécessaire, puisque Susanoo englobe à présent sa fonction. Ainsi, il s'unit à Kushi inada hime, puis à Kamu oho ichi hime no kami, dont il obtient deux enfants :

Oho toshi no kami et Uka no mitama no kami. ${ }^{12}$

Si le second représente simplement la divinité âme (mitama) de la nourriture (uka) et ne sera que peu présent dans les rituels futurs, le premier enfant est beaucoup plus complexe et aura une présence essentielle dans les traditions populaires, puisque considéré par la suite comme un avatar du yama no kami.

31 En effet, s'il faut comprendre le terme oho (qui s'oppose à celui de wakai en japonais) comme quelque chose d'accompli (arrivé au stade ultime de son évolution), le terme toshi a certes comme en japonais moderne la valeur sémantique d'année, mais aussi par extension celle de découpage calendaire, par rapport au calendrier lunaire. Pourtant, ce même toshi peut aussi revêtir le sens de céréale (de riz qui est la céréale par excellence au Japon) lorsqu'il devient la lecture du caractère ine (ina).

Il est à noter qu'on retrouve encore dans les rites agricoles (Miyamoto Tsune'ichi et même Yanagida Kunio en ont tous deux parlé dans leurs essais) un Messire Toshigami (Toshigami sama), appelé parfois Shogatsu sama (Messire nouvel an (du calendrier lunaire)) et qui est comme nous l'avons déjà dit une représentation du dieu de la montagne (le protecteur des récoltes).

Ainsi, au début de l'année lunaire retrouvera-t-on accrochées aux portes des fermes des bûches de bois appelées saigi (arbre du bonheur) ou toshigi (arbre du riz), ou bien, planté devant les demeures, un bambou au sommet duquel est placé un panier destiné à recevoir le dieu. Par ce dernier rite, on demande au dieu de redescendre de la montagne et de reprendre sa fonction de dieu des champs.

Mais on peut aller encore un peu plus loin dans l'analyse du nom de ce dieu Oho toshi, lorsqu'on se réfère à l'expression, d'ailleurs encore usuelle en japonais moderne, de otoshi dama. Celle-ci désigne de nos jours les étrennes d'argent qu'on donne aux enfants. Or, à l'origine, on offrait aux enfants comme à ses supérieurs, des mochi (pâte de riz cuit à la vapeur et pilonné), des oho hineri (riz enveloppé dans du papier en forme de boules) ou des nigiri motchi (boule de mochi). Toutes ces offrandes étaient donc autant de formes de boules, tama en japonais, cette forme étant dès le Japon proto-historique la représentation de l'âme tamashi. 


\section{déesse de la nourriture abond
l'ordre suivant leurs enfants}

- Waka yama ku hi no kami (Kami de la jeune montagne) représente une divinité au pied de la montagne, donc le dieu serpent qui revient prendre sa fonction de dieu des champs.

- Waka toshi no kami (Kami du riz jeune) s'oppose à Oho toshi et représente les pousses de riz qui seront à replanter.

- Wakasaname no kami (déesse du repiquage du jeune riz).

- Mizumaki no kami (divinité de l'eau).

- Natsu taka tsuhi no kami (dieu du soleil de l'été).

- Natsu no me no kami (déesse de l'été).

- Akibime no kami (déesse de l'automne).

- Kukutoshi no kami (dieu des céréales en tiges).

- Kukuki wakamuro tsunane no kami (dieu des nouvelles demeures construites de tiges encordées).

Pour résumer ce que nous venons d'écrire sur les différents récits mythiques abordés, nous pouvons déduire que Susanoo, loin d'être un dieu destructeur, asocial et violent tel que décrit le plus souvent, est au contraire très clairement à l'origine des semences (donc de la nourriture). En outre, c'est également lui qui met en route le cycle calendaire agraire.

Sa fonction est donc primordiale et éclipse de loin la fonction de la divinité solaire.

41 Mais nous voudrions revenir avant de conclure sur un passage du mythe du meurtre sacrificiel de la déesse Oho getsu hime par Susanoo. Dans ce mythe, on assiste au démembrement rituel de la déesse : des morceaux de son corps apparaissent les céréales.

Or, ce démembrement nous renvoie à un récit à propos de Yamato Takeru ( 0 usu no mikoto) (fils renié de l'Empereur mythique Keikô) qui tue son jumeau, mais aîné, Oho usu no mikoto ${ }^{14}$. Dans ce dernier cas, le lien à l'agriculture (voire à la nourriture) est beaucoup plus mince et seul l'emploi du terme usu, présent dans le nom des deux protagonistes, permet de l'établir, puisque usu signifie le mortier servant à écraser le riz.

43 La nécessité rituelle du meurtre et du démembrement narrés semble s'expliquer par le banquet qui suit et qui précède un départ pour la guerre.

Oetrouve d'ailleurs ce même motif du meurtre et du démembrement lié au banquet dans les conquêtes du premier empereur mythique l'Empereur Jimmu. Il concerne les frères Ukashi que l'empereur veut soumettre ${ }^{15}$. Le cadet vient faire sa soumission et dire à l'Empereur que son frère aîné a préparé un piège pour le faire périr. Mais finalement, 
c'est lui qu'on force à tomber dans son propre piège et il est démembré. Le cadet offre alors un banquet. cultuel du Japon, alors même qu'au cours de l'histoire culturelle de l'archipel bien des croyances autochtones ou étrangères s'y sont greffées, jusqu'à en atténuer (voire se substituer) le sens primordial.

${ }^{17}$ les parents mangent le repas et se retrouvent bloqués dans un autre monde, ils procèdent du même mythème que lorsqu'Izanami, ayant mangé le repas du Pays de Yomi, ne peut revenir dans le Pays au milieu de la Plaine de Roseaux (Ashi hara no nakatsu kuni). ${ }^{18}$

\section{NOTES}

1. Les mochi sont des gâteaux de riz pilé qui peuvent être sucrés ou salés et qui ont une consistance pour le moins molle et élastique.

2. Les veillées avaient traditionnellement lieu le soir, mais de nos jours, pour des raisons pratiques, elles se déroulent le lendemain du décès. 
3. Le Kojiki est un ouvrage de mythes et de récits des empereurs mythiques, légendaires puis historiques dont la compilation fut achevée en 712 . Nous utilisons pour notre étude l'édition japonaise suivante: Kojiki (Chronique des faits anciens), 1, Nihon koten bungaku zenshû, Shogakkan, 1997.

4. Le Nihonshoki est, comme le Kojiki, un ouvrage de mythes et de récits des empereurs mythiques, légendaires puis historiques, mais dont la compilation fut achevée en 720 . Nihonshoki (Écrits du Japon), 2,3 et 4, Nihon koten bungaku zenshû, Shogakkan, 1997.

5. Sur ces rites associés au môgari, on se reportera à François Macé, La mort et les funérailles dans le Japon ancien, POF, 1986.

6. Il s'agit du Mythe du serment (Ukehi no shinwa) et du Mythe de la caverne (Ame no Iwayato no shinwa) et qui narrent pour le premier une hiérogamie incestueuse et pour le second la réclusion de la déesse du soleil Amaterasu dans une caverne (mort symbolique) puis sa re-naissance.

7. Ibid., pp. 68-69.

Il est à noter sur ce passage que le Nihonshoki laisse apparaître d'autres choses dans un ordre distinct de celui proposé par la version du Kojiki (dont gibier, bœufs et chevaux). D'autre part, Ukemochi (divinité des aliments) y est tuée par Tsukuyomi (divinité de la lune). Mais le récit garde une même structure et Susanoo et Tsukuyomi forment un couple, ou plutôt on peut affirmer que Tsukuyomi est une part de Susanoo qui transcende les forces du jour, comme celles de la nuit.

8. Ibid., pp. 40-44.

9. Sur l'évolution de ces procédés rituels, les travaux exemplaires de Yoshino Hiroko nous montrent l'émergence et la complexité des syncrétismes entre le fond rituel shintô (qui provient déjà lui-même de sources et de pratiques indigènes et étrangères) et les idées neuves du taoïsme, qui devint très à la mode à l'époque de l'Empereur Tenmu.

10. Entre autres liens symboliques à la mort, on notera que l'entrée d'un des trois domaines post mortem du Japon (et le plus mortifère), le Yomi no kuni (le Pays de Yomi) s'y trouve (il s'agit de la Descente Yomotsu hirasaka). De nombreux essais traitent de cette fonction; on pourra se reporter à Kanda Norishiro, Kodai Izumo to shisha no sekai (L'Ancien Izumo et le monde des morts), Tairikushobô, 1986.

11. Ibid., pp. 68-72.

12. Ibid., pp. 72-75.

13. Ibid., pp. 96-99.

14. Ibid., pp. 215-216.

15. Ibid., pp. 150-152.

16. On pourra voir par exemple : Yoshida Atsuhiko, Jômon no shinwa (Les mythes de l'époque de Jômon), Seidosha, 1987 et Yoshida Atsuhiko, Nihon shinwa no tokushoku (Les spécificités de la mythologie japonaise), Seidosha, 1989.

17. Célèbre film d'animation japonais réalisé par Miyazaki Hayao en 2001, sorti en France sous le titre Le voyage de Chihiro, mais dont le titre japonais était: Sen to Chihiro no kami kakushi (L'enlèvement par les kami de Sen et de Chihiro).

18. Ibid., pp. 44-48. 


\section{RÉSUMÉS}

Le propos de notre article est de mettre en relation les motifs proposés par les mythes japonais et les rites alimentaires pratiqués lors de la veillée funéraire. Nombreux sont les récits mythiques où le mythème de l'alimentation est évident, quand il ne se révèle pas essentiel lors d'une lecture palimpseste. Le dieu Taka haya Susaoo no mikoto (frère de la grande déesse du soleil Amaterasu oho mi kami) est considéré le plus souvent par les chercheurs comme une entité asociale et destructrice; or, tout au contraire, nous cherchons à montrer qu'il est indispensable au processus mythique, lié à la genèse de l'agriculture, et donc à celle des habitudes alimentaires de l'archipel. Ainsi, pour les dieux (kami) comme pour les défunts ou les (sur)vivants d'hier à aujourd'hui, les pratiques alimentaires se retrouvent-elles liées à un imaginaire chthonien, psychopompe et cultuel inscrit, pour partie dans les textes, et pour partie dans les mémoires (inconscientes) des pratiques collectives.

\section{AUTEUR}

\section{JEAN-PIERRE GIRAUD}

Jean-Pierre Giraud est professeur à l'Université Lyon Jean Moulin, où il dirige le département d'études japonaises depuis 2001. Membre de l'Institut des Etudes Transtextuelles et Transculturelles (IETT) de Lyon III, ses travaux portent sur l'imaginaire religieux et culturel du Japon. Parmi ses publications, on peut noter la direction du dossier : Imaginaires féminins : Japon (Iris numéro 30, CRI, Grenoble III, 2007) et la co-direction avec Christian Galan (Toulouse II) de l'ouvrage collectif Individu-s et démocratie au Japon (aux Presses universitaires du midi, 2015). 